\title{
Delayed massive bleeding caused by an ingested
} fish bone

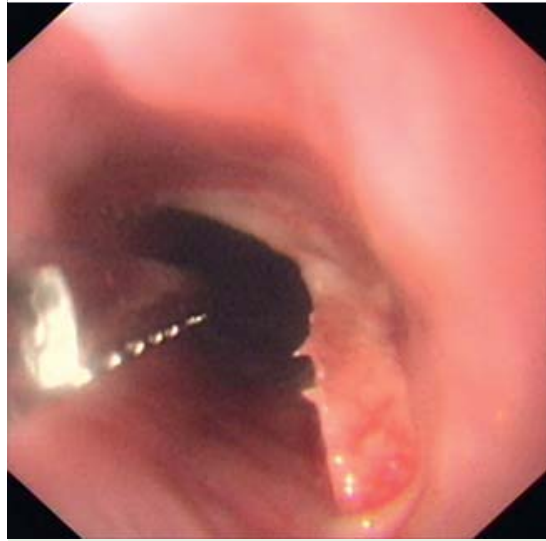

Fig. 1 Endoscopic view of a fish bone embedded in the esophagus of a 48-year-old man presenting to the hospital because of chest pain without hematemesis or melena.

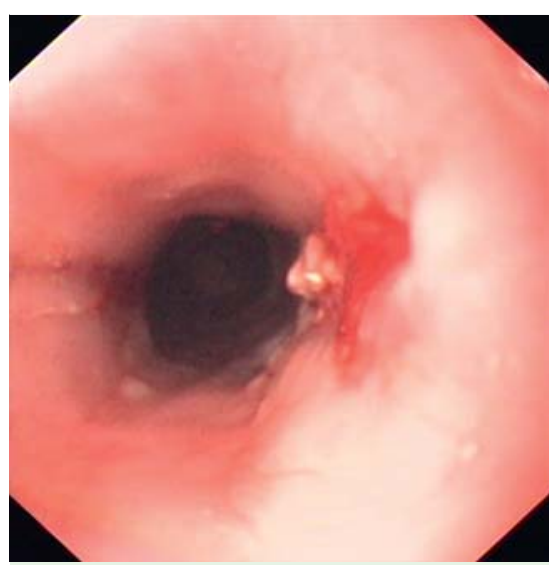

Fig. 3 Oozing bleeding at the wound after removal of the fish bone.

A 48-year-old man came to our hospital after having ingested a fish bone 2 days earlier; he was experiencing chest pain without hematemesis or melena. Esophagogastroduodenoscopy revealed a fish bone embedded in the esophagus at approximately $27 \mathrm{~cm}$ from the incisors ( $\bullet$ Fig. 1). Endoscopic removal of the bone was successful ( $\bullet$ Fig. 2), and oozing bleeding at the wound was noticed ( $\bullet$ Fig.3). The patient was asked to remain in the hospital for at least 2 days while on a liquid diet, but he refused and went back home.

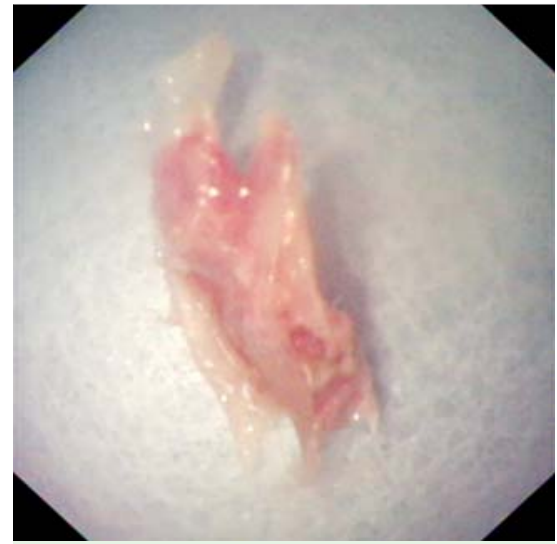

Fig. 2 The removed fish bone.

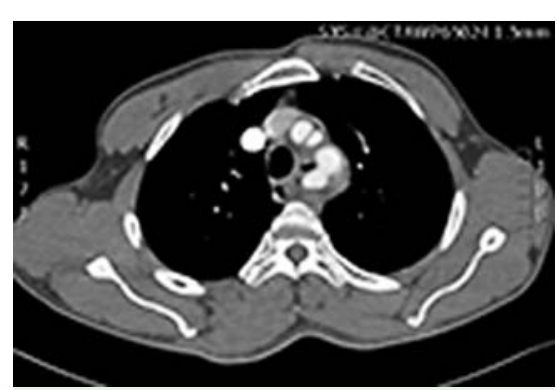

Fig. 4 Computed tomography reveals a mediastinal abscess and rupture of the aortic arch with the formation of a pseudoaneurysm.

A week later, he presented to the emergency room with massive hematemesis. Emergency computed tomography of the chest revealed a mediastinal abscess and rupture of the aortic arch with the formation of a pseudoaneurysm ( Fig.4, - Video 1). The patient died of a massive hemorrhage before emergency surgery could be performed.

Foreign body ingestion occurs commonly, and in the majority of cases, the body can pass spontaneously once it gets through the esophagus [1]. However, sharp, pointed objects, such as fish bones and toothpicks, may lodge in the esophagus and increase the risk for complications such as perforation, hemorrhage, aortoesophageal fistula, and even death; therefore,

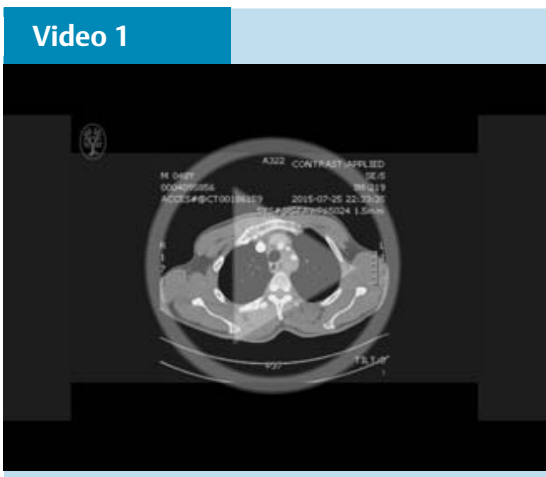

Computed tomography reveals a mediastinal abscess and rupture of the aortic arch with the formation of a pseudoaneurysm.

emergency endoscopic intervention is required [2,3]. In the present case, the fish bone had lodged in the patient's esophagus for 2 days before being successfully removed endoscopically. However, delayed bleeding developed a week after endoscopic removal, and the patient died of a massive hemorrhage.

Two lessons can be learned from this case. First, if a sharp foreign body has lodged in the esophagus for more than 24 hours and has become embedded, it is prudent to perform computed tomography before endoscopic removal to check for the absence of any complication and assess the relationship of the foreign body to the airway and vessels. Second, careful observation in the hospital is recommended for any patient who has undergone endoscopic removal of an embedded foreign body.

Endoscopy_UCTN_Code_CPL_1AH_2AJ

Competing interests: None

\section{Yuyong Tan, Yi Chu, Deliang Liu, Jirong Huo}

Department of Gastroenterology, The Second Xiangya Hospital of Central South University, Hunan, China 


\section{References}

1 Webb WA. Management of foreign bodies of the upper gastrointestinal tract: update. Gastrointest Endosc 1995; 41: 39-51

2 Ikenberry SO, Jue TL, Anderson MA et al. Management of ingested foreign bodies and food impactions. Gastrointest Endosc 2011; 73: $1085-1091$

3 Yamada T, Sato H, Seki $M$ et al. Successful salvage of aortoesophageal fistula caused by a fish bone. Ann Thorac Surg 1996; 61: $1843-1845$

\section{Bibliography}

DOI http://dx.doi.org/

10.1055/s-0034-1393379

Endoscopy 2015; 47: E569-E570

(c) Georg Thieme Verlag KG

Stuttgart · New York

ISSN 0013-726X
Corresponding author

\section{Jirong Huo, MD}

Department of Gastroenterology

The Second Xiangya Hospital of Central South University

No. 139 Middle Renmin Road

Changsha

Hunan, 410011

China

Fax: +86-0731-85533525

hjr198@hotmail.com 